\title{
Las relaciones laborales en la economía de plataformas
}

\author{
Holm-Detlev Köhler ${ }^{1}$ \\ Recibido 11/02/2020; Aceptado 03/04/2020
}

Resumen. El objetivo del presente artículo consiste en desarrollar un marco analítico y conceptual para el análisis de la acción colectiva y las prácticas organizativas de los trabajadores de plataformas. Para esto recurrimos a enfoques clásicos de la sociología del trabajo y a la teoría de las fuentes de poder sindical. La base empírica son estudios y encuestas recientes del trabajo de plataformas, el análisis de páginas web de acción colectiva y conversaciones con activistas de las plataformas. Se examina las características específicas de la acción colectiva y de las prácticas organizativas de los trabajadores de plataformas para identificar su potencial y las oportunidades en la perspectiva de posibles fuentes de poder sindical.

Palabras clave: Sociología del trabajo de plataformas; Economía de plataformas; Fuentes de poder sindical; Relaciones laborales.

\section{[en] Labour relations in the platform economy}

Abstract. The aim of this paper is to develop a conceptual framework for analysing the collective actions and organisational practices of platform workers, building on classical approaches of the sociology of capitalist work and on the power resources theory employed in current trade union analysis. The empirical bases are recent surveys and studies on platform work, the analysis of websites for the collective action of platform workers and conversations with platform activists. The specific characteristics of platform workers' collective actions and organisational practices are examined with a view to identifying their potential and the opportunities they afford in the light of different trade union power sources.

Keywords: Sociology of platform work; Platform economy; Power sources; Employment relations.

Sumario: Introducción. La sociología del trabajo de plataformas. Las relaciones laborales en la economía de plataformas. Las fuentes de poder de los trabajadores de plataformas. Conclusiones. Bibliografía.

Cómo citar: Köhler, H-D. Las relaciones laborales en la economía de plataformas. Sociología del Trabajo, nº96 (2020), 23-33.

\section{Introducción}

Not so long ago, the only people who looked for "Gigs" were musicians. For the rest of us, once we outgrew our school dreams of rock stardom, we found "real" jobs that paid us a fixed salary every month, allowed us to take paid holidays and formed the basis for planning a stable future (Arun Sundararajan, The Guardian, 26/07/2015).

En la reunión anual del Foro Económico Mundial en Davos 2020, varias compañías líderes de plataformas, incluidas Uber, Deliveroo y Cabify, publicaron la 'Carta de principios para un trabajo decente de plataformas' como reacción a la creciente conciencia pública de los problemas sociales y laborales de su modelo de negocio. La economía de plataformas emergente está remodelando los modelos de negocio y las relaciones laborales en Europa, desafiando a los agentes sociales convencionales y las instituciones reguladoras. "Economía de plataformas" o "gig economy" se refiere a plataformas digitales que coordinan la demanda de servicios específicos con proveedores de servicios indi-

Departamento de Sociología de la Universidad de Oviedo

hkohler@uniovi.es 
viduales utilizando algoritmos digitales. Las plataformas de trabajo digital son agentes económicos que proporcionan espacios virtuales para ajustar la oferta y la demanda de trabajo a través de tecnologías en red basadas en la gestión algorítmica, lo que reduce sustancialmente los costos de transacción. En la teoría económica clásica de la empresa, los costos de transacción son la explicación principal de la existencia de la empresa como organización en una economía de mercado (Coase 1937). El empresario clásico era un tomador de riesgo que movilizaba capital de riesgo. El propietario de la plataforma, por el contrario, transfiere casi todos los riesgos y costos a otros agentes externos. Al eliminar así la razón clave de la existencia de organizaciones de generación de valor, "las plataformas en red empujan el proceso de descentralización, creación de redes, subcontratación, y parcelación del trabajo en micro-tareas o ' $g i g s$ ' hacia un nuevo límite en el que todo lo que queda de la empresa es una técnica para obtener beneficios" (Visser 2019, 9; traducción propia). "La 'gestión algorítmica' permite a estas plataformas rastrear y disciplinar cada vez más a los trabajadores, en muchos casos eludiendo o incumpliendo las regulaciones laborales y de salud e higiene existentes, en detrimento de la protección social y personal de los trabajadores" (Vandaele 2018, 5; traducción propia). Las tecnologías digitales están dando lugar a un nuevo modelo de negocio anonimizando las relaciones entre empleadores, empleados autónomos y clientes, desafiando así los sistemas tradicionales de regulación institucional. Estas empresas externalizan todas las relaciones con clientes y empleados, maximizando así la desinstitucionalización y la flexibilización de la prestación de servicios, la jornada laboral y las relaciones laborales. En particular, la economía de plataformas desafía a todos los mecanismos de organización y representación colectiva conseguidos por la clase obrera en el curso del siglo XX en su lucha por civilizar las economías capitalistas y decomodificar el trabajo. En este artículo analizamos los intentos emergentes de los trabajadores de plataformas para desarrollar capacidades de acción colectiva y estrategias de resistencia.

El artículo responde a la siguiente estructura. La siguiente sección examina las características principales del trabajo de plataformas recurriendo a los enfoques sociológicos para conceptualizar la organización del trabajo capitalista. La tercera sección se basa en evidencias empíricas y experiencias de acción colectiva de los trabajadores de plataformas para analizar la estructura particular de las relaciones laborales y de la acción colectiva. Las bases empíricas son encuestas y estudios recientes sobre el trabajo de plataformas, análisis de páginas web para la acción colectiva de los trabajadores de plataformas y conversaciones con activistas en España, Alemania, el Reino Unido y Noruega. La cuarta sección examina el potencial y las oportunidades de las acciones colectivas de los trabajadores de plataformas a la luz de las diferentes fuentes de poder sindical. El artículo se cierra con una breve reflexión concluyente.

\section{La sociología del trabajo de plataformas}

El trabajo de plataformas, la organización de la oferta y de la demanda de mano de obra remunerada a través de una plataforma en digital, es una forma de empleo emergente y en crecimiento que aún careciendo de una definición clara, muestra una heterogeneidad de modelos de negocio y requiere una regulación del contrato de trabajo, del régimen de empleo y de las condiciones de trabajo (Mandl 2019). Esta categoría incluye tanto plataformas basadas en una web, donde el trabajo se subcontrata a través de una convocatoria abierta a una multitud dispersa geográficamente (“crowdwork"), como aplicaciones localizadas que asignan el trabajo a personas en un área geográfica específica.

Tabla 1: Cinco tipos de trabajo en plataformas

\begin{tabular}{|l|l|l|}
\hline \multicolumn{1}{|c|}{ Tipo } & \multicolumn{1}{|c|}{ Descripción } & \multicolumn{1}{c|}{ Ejemplos } \\
\hline $\begin{array}{l}\text { Plataformas locales de trabajos ruti- } \\
\text { narios }\end{array}$ & $\begin{array}{l}\text { La plataforma asigna tareas específi- } \\
\text { cas a personas en un área local }\end{array}$ & $\begin{array}{l}\text { Servicios de transporte y de comida a } \\
\text { domicilio como Uber y Deliveroo }\end{array}$ \\
\hline $\begin{array}{l}\text { Plataformas de demanda para trabajos } \\
\text { específicos }\end{array}$ & $\begin{array}{l}\text { Los clientes eligen trabajadores para } \\
\text { tareas específicas }\end{array}$ & $\begin{array}{l}\text { Plataformas de servicio de tareas do- } \\
\text { mésticas como Oferia }\end{array}$ \\
\hline $\begin{array}{l}\text { Plataformas locales de trabajadores } \\
\text { cualificados }\end{array}$ & $\begin{array}{l}\text { Los trabajadores ofrecen servicios de } \\
\text { trabajo específico }\end{array}$ & $\begin{array}{l}\text { Plataformas de servicio de trabajos } \\
\text { domésticos como ListMinut }\end{array}$ \\
\hline $\begin{array}{l}\text { Plataformas de trabajo online (click- } \\
\text { work) }\end{array}$ & $\begin{array}{l}\text { La plataforma asigna trabajos a perso- } \\
\text { nas para su realización online }\end{array}$ & $\begin{array}{l}\text { Plataformas de servicios profesionales } \\
\text { como Crowdflower }\end{array}$ \\
\hline $\begin{array}{l}\text { Plataformas de servicios especializa- } \\
\text { dos online }\end{array}$ & $\begin{array}{l}\text { Los trabajadores compiten online } \\
\text { realizando trabajos cualificados y el } \\
\text { cliente selecciona al ganador }\end{array}$ & $\begin{array}{l}\text { Plataformas de servcios profesionales } \\
\text { como 99designs }\end{array}$ \\
\hline
\end{tabular}

Fuente: Mandl 2019, 6

Las principales diferencias entre estos tipos residen en la escala de tareas, la prestación del servicio (online o local), las cualificaciones requeridas, el proceso de contacto entre clientes y trabajadores y la forma de asignación de trabajo. Desde el punto de vista de la sociología del trabajo, la economía de plataformas representa una forma nueva y específica de organización del trabajo capitalista en varias dimensiones. 
Siguiendo teorías clásicas sobre el desarrollo capitalista, la economía de plataformas es una nueva forma de colonización capitalista de espacios no o pre-capitalistas. Rosa Luxemburg (1912; véase también David Harvey 2003) criticó a los esquemas de reproducción capitalista de Marx (volumen II de El Capital), basados exclusivamente en los propietarios de capital y la fuerza laboral, como demasiado cerrados y estáticos. Para poder explicar el dinamismo del capitalismo se requiere un concepto de reproducción ampliada donde el capital incorpora e invade constantemente esferas no capitalizadas.

La acumulación capitalista necesita, para su desarrollo, un medio ambiente de formaciones sociales no capitalistas; va avanzando en constante cambio de materias con ellas, y sólo puede subsistir mientras dispone de este medio ambiente (Luxemburg 1912, 281).

Luxemburg distinguió dos formas de colonización de espacios no capitalistas o pre-capitalistas: colonización externa hacia países y regiones pre-capitalistas y colonización interna de esferas no capitalistas en las economías capitalistas existentes. La economía de plataformas combina ambas formas de colonización capitalista. El trabajo de plataformas abre nuevos espacios para la movilización capitalista de mano de obra barata y flexible, operando en nuevos espacios hasta ahora fuera del alcance del capital. Visto desde esta perspectiva, el trabajo de plataformas es la colonización para la explotación capitalista de nuevos espacios de vida (tardes, fines de semana de estudiantes, amas de casa, trabajadores de cuidado, trabajadores rurales en África o Asia, etc.) para la producción de valor capitalista. Las redes digitales facilitan el acceso extendido temporal y espacialmente a grupos de trabajadores que de otro modo serían inaccesibles para el trabajo asalariado (Altenried 2020). Así, por ejemplo, los trabajadores en red en África o Asia a menudo tienen que trabajar de noche para estar sincronizados con las zonas horarias de sus clientes en América del Norte o Europa Occidental (Wood et al. 2019, 67).

Desde una perspectiva histórica del capitalismo, muchas, si no todas, las prácticas de organización de trabajo de las plataformas no son realmente novedosas (Vandaele 2018; Daugareilh et al.2019). Descomponer los trabajos en tareas pequeñas, poco cualificadas y repetitivas, prácticas de producción doméstica, talleres rurales domésticos ('putting out' system), trabajo a pedido, trabajo a destajo, los modelos de negocio basados en intermediarios, etc., formaban una parte integral del capitalismo temprano en Europa occidental hasta el siglo XIX y siguen siendo común en los países en vías de desarrollo hasta hoy. La economía de plataformas está reintroduciendo estas prácticas en los países centrales occidentales, al mismo tiempo que permite la explotación de las diferencias geográficas en cualificaciones, costos laborales, regulaciones ambientales y fiscales.

El trabajo de plataformas representa una nueva forma de trabajo capitalista, una nueva dimensión de la recomodificación del trabajo en el contexto de la hegemonía neoliberal a partir de la década de los 1970, un nuevo terreno disputado para ser regulado por la política, la legislación laboral, los grupos de interés y los agentes sociales, etc., un desafío para inventar nuevos entornos institucionales en un campo emergente de trabajo precario y flexible (Dueñas Herrero 2019). En gran medida, es un trabajo poco calificado, flexible pero estandarizado, fuera de las relaciones laborales regulares, una especie de taylorismo digital sin el trabajador en masa taylorista (Altenried 2020; Howcraft y Bergvall-Kåreborn 2019). Como nuevo modelo de negocio, la empresa app representa la desintegración completa de la organización tradicional fordista y sus grupos de interés en un organismo flexible, impulsada por el mercado e individualizada en forma de red de empresa-cliente-trabajador. La estructura institucional construida alrededor de la empresa fordista con una fuerza laboral contractual fija, negociación colectiva y relaciones laborales, leyes laborales, seguridad social, salud e higiene, responsabilidades fiscales y responsabilidad social se está desvaneciendo.

El clásico problema de transformación en la gestión de la mano de obra, es decir, cómo convertir la fuerza laboral contratada en trabajo efectivo para la creación de valor (Köhler y Martín Artiles 2010, 102), adopta una nueva forma de control gerencial en el caso del trabajo de plataformas. Todo el control directo y personal es reemplazado por una app que ejerce una vigilancia total sobre los trabajadores a través de mensajes automáticos, asignación de tareas, determinación del tiempo de trabajo, control de ubicación, evaluación del desempeño (calificación y clasificación), etc . "Un Panóptico algorítmico proporciona una visión divina sobre el comportamiento de los trabajadores a través de una combinación de taylorismo y panopticismo" (Ivanova et al. 2018, 13; traducción propia). El incesante proceso de evaluación y clasificación genera un nivel de presión completamente fuera de sincronía con la actividad o tarea ejecutada (Howcraft y Bergvall-Kåreborn 2019, 30). La app es el jefe real y está totalmente en manos del empleador, lo que representa la expropiación más completa de los medios de producción de los trabajadores en la era capitalista.

Para muchos neoliberales, estos movimientos hacia una flexibilidad total para el capital alcanzan su apogeo en el desarrollo de los llamados 'contratos de hora cero' en los que los trabajadores no tienen garantizado ningún trabajo, pero deben estar disponibles 'de guardia' cuando el empleador los necesita (Herodes y Lambert 2016, 22). "Es una pena que Karl Marx no estuviera presente... El autor del Manifiesto Comunista tendría mucho que decir sobre la noticia de que según estimaciones oficiales esta forma de trabajo informal se habían disparado en un $25 \%$... Se habría burlado de aquellos que ven en los contratos de cero horas una expresión de la fortaleza económica de Gran Bretaña, una demostración de mercados laborales flexibles en acción. Habría pensado que el 'ejército de reserva de trabajo' era una mejor descripción de las condiciones en las que se esperaba que los trabajadores estuvieran permanentemente de guardia para un empleador.” (Larry Elliot en The Guardian, 04/08/2013; traducción propia) 


\section{Las relaciones laborales en la economía de plataformas}

Las plataformas laborales digitales tienden a eludir las normas existentes sobre empleo, protección social e impuestos corporativos. Trabajar en este sector puede ser extremadamente aislado, con la app como único canal de comunicación entre el trabajador y el empleador y sin comunicación entre los empleados. La organización y la acción colectiva son muy poco probables en estas condiciones. Los empleadores tienden a negar sus responsabilidades al no reconocer a sus trabajadores como empleados ni a ellos mismos como empleadores. Los que trabajan para ellos son considerados trabajadores autónomos o 'contratistas independientes'.

Los trabajadores ya no son técnicos, conductores u operadores internos, sino proveedores de servicios externos que realizan su trabajo dentro de la empresa sin pertenecer a ella, sin conocer a sus colegas, sin tener voz en la organización del trabajo, sin conocer al gerente de Recursos Humanos o el jefe del departamento para el que están trabajando, sin contactar a los representantes sindicales, sin discutir las cosas con sus compañeros tomando un café. Aunque trabajan para la empresa, solo tienen un papel marginal ... La transformación (o hibridación) de una empresa tradicional en una plataforma digital significa nada menos que el abandono de todo el campo de las relaciones laborales por parte del empresario. Una plataforma no es más que un mercado de servicios, en el que no hay lugar para las leyes laborales ni para la seguridad social (Daugareilh et al.2019, 21, 27; traducción propia).

La mayoría de los trabajadores de plataformas están subempleados con bajos salarios y malas condiciones de trabajo, complementando los ingresos de otros trabajos o combinando el trabajo doméstico con el trabajo de la plataforma. Las mujeres en particular combinan el crowdwork con las responsabilidades de cuidado (OIT 2018). Los trabajadores de plataformas a menudo no llegan al salario mínimo, carecen de todos los derechos laborales elementales, como vacaciones pagadas, pago en case de enfermedad, seguro en caso de accidentes o enfermedades, seguridad social y tienen que pagar su propio medio de transporte (bicicleta, moto) y sus teléfonos móviles.

A pesar de las relaciones laborales individualizadas y las dificultades para organizarse colectivamente, "muchos trabajadores (28 a 60 por ciento, dependiendo de la plataforma encuestada) han recurrido a foros online y redes sociales dirigidos por trabajadores para obtener asesoramiento o participar en debates sobre los problemas que enfrentan los trabajadores de plataformas"(OIT 2018, xviii). Con respecto a las relaciones laborales, deben distinguirse al menos dos tipos diferentes de plataformas de trabajo. Las plataformas de reparto y transporte como Deliveroo o Uber ofrecen servicios locales por parte de trabajadores localizados, mientras que las plataformas globales de Internet como Amazon Mechanical Turk o Upwork brindan sus servicios a escala global y permiten que el trabajo se descomponga en muchas micro tareas distribuidas por todo el mundo. Aunque resulta difícil, es posible organizar los primeros y, de hecho, cada vez hay más intentos de riders y conductores de organizarse colectivamente (ver más abajo). Con respecto a los segundos, es extremadamente difícil reunir a estos trabajadores en torno a intereses compartidos. Por lo tanto, en este artículo nos centramos principalmente en las plataformas de reparto y transporte.

Siguiendo los análisis del trabajo de plataformas por parte de observatorios internacionales y de sindicatos (UGT 2019; OIT 2018; Perera et al. 2020), las características principales del trabajo de plataformas de reparto son:

a. El rider no tiene voz ni influencia en el contenido del contrato impuesto por el empleador.

b. El rider es siempre y fácilmente sustituible a través de la simple suspensión unilateral del contrato.

c. El empleador determina todas las condiciones de trabajo, el precio, el tiempo y la forma de entrega sin negociación ni consulta.

d. El empleador tiene una amplia gama de trabajadores a su disposición, trabajadores sin ningún poder de negociación o infraestructura organizativa.

El trabajo de plataformas implica, por lo tanto, una estrategia del empleador de traslado de todos los riesgos y responsabilidades de la relación laboral a los trabajadores. Para los sindicatos, estas características implican varios desafíos clave para organizar y sindicalizar a los trabajadores de este sector:

1. La falta de un contrato de trabajo claramente definido (muchos trabajan por cuenta propia) hace que la representación y organización en instituciones tradicionales de relaciones laborales sea muy difícil.

2. Los trabajadores no se reúnen en un centro de trabajo común, no comparten espacios físicos, lo que complica la comunicación y la organización colectiva.

3. El trasfondo cultural y educativo de muchos trabajadores, a menudo jóvenes con niveles educativos altos, implica una cierta distancia a la representación sindical tradicional.

4. El estado desprotegido de los trabajadores, expuesto todo el tiempo a un reemplazo fácil con solo suspender las órdenes, y su falta de poder de negociación efectivo hace que la sindicación sea improbable.

Un Deliveroo rider de Barcelona explica su situación (UNO, 22/07/2017, http://www.unologistica.org/riders-X-derechos-urge-regular-la-figura-del-repartidor-bici-la-economia-colaborativa/): 
La manera de llevar el tema de nuestra autonomía de Deliveroo es realmente agobiante. El no poder controlar tu situación cuando realmente estás pagando unos gastos para poder controlarla. Otra cosa que afecta mucho es el hecho que te comentan unas cosas cuando vas a empezar que resultan falsas, como que no harás un pedido de más de 6 kilómetros o que estarás protegido en condiciones de climatología extremas y no es cierto. No es posible que no haya un plus si pasa el kilometraje o conduciendo bajo una tormenta. El mayor inconveniente es lo vulnerable que somos en esta situación de falsos autónomos, física y laboralmente.

\section{Un compañero de Londres añade:}

Al principio escribí correos electrónicos, correos largos, señalando formas en que la plataforma podría funcionar mejor para los trabajadores. Con capturas de pantalla, explicaciones detalladas de cómo nos estaban haciendo la vida difícil, pero me di cuenta de que no les importa eso. Si les planteas algún problema, simplemente te despedirán o encontrarán una manera de dejar de darte trabajo (Perera et al. 2020, 14; traducción propia).

La mayoría de las plataformas, incluidas Uber, Amazon Mechanical Turk, TaskRabbit y Upwork, son empresas transnacionales. Las apps de reparto de comida Deliveroo y Foodora tenían alrededor de 50,000 y 7,000 riders trabajando para ellos en 13 y 10 países respectivamente en 2018 (Ivanova et al. 2018). Aunque la organización y la acción colectiva son desafíos difíciles para los trabajadores de plataformas y los sindicatos, hay un número creciente de experiencias alentadoras de luchas auto-organizadas de los trabajadores e iniciativas sindicales para apoyarles (ver también Degryse 2016).

Una forma de apoyo sindical es el establecimiento de una página web específica para los trabajadores. La iniciativa más completa y avanzada hasta ahora es la plataforma Fair Crowdwork (http://www.faircrowdwork.org/), un proyecto conjunto de IG Metall (el Sindicato de Trabajadores Metalúrgicos de Alemania), la Cámara de Trabajo de Austria, la Confederación de Sindicatos de Austria y el sindicato de cuello blanco sueco Unionen, en asociación con varios expertos e investigadores. Los días 13 y 14 de abril de 2016, esta red celebró el primer Taller Internacional sobre Estrategias Sindicales en la Economía de Plataformas en Frankfurt am Main (Alemania), que reunió a sindicalistas de las organizaciones mencionadas, junto con expertos jurídicos y técnicos de Asia, Europa y América del Norte. Ahí se aprobó la "Declaración de Frankfurt" (https://www.igmetall.de/download/20161214_Frankfurt_Paper_on_Platform_Based_Work_EN_b939ef89f7e5f3a639cd6a1a930feffd8f55cecb.pdf) para un trabajo justo basado en las plataformas. En un proceso de aprendizaje interactivo, Fair Crowdwork amplía continuamente su alcance y sus actividades. La confederación sindical española UGT (Unión General de Trabajadores), por su parte, lanzó en 2017 la página web http://turespuestasindical.es/ como un servicio para trabajadores de plataformas, ofreciendo asesoramiento y asistencia legal de expertos, contacto con redes y servicios complementarios.

Cada vez más federaciones sindicales en toda Europa, como el sindicato austríaco de servicios privados GPA-djp (Gewerkschaft der Privatangestellten, Druck, Journalismus, Papier) están ofreciendo la afiliación con una tarifa plana a los trabajadores de plataformas incluyendo protección legal y asesoramiento. En el suroeste de Francia (Gironda/ Burdeos), el sindicato CGT creó una federación de mensajería en bicicleta, que representa a cientos de riders de reparto. En el Reino Unido, el Sindicato Independiente de Trabajadores de Gran Bretaña (IWGB), una escisión de Unite y UNISON que organiza sobre todo a trabajadores migrantes, ha apoyado varias campañas y huelgas de riders de reparto que reclaman el estatus de empleados.

Hasta ahora los convenios colectivos en el sector de plataformas digitales son una rara excepción. En Dinamarca, la Federación Unión de Trabajadores Daneses concluyó en 2018 un convenio colectivo piloto de 12 meses con la plataforma digital del sector de la limpieza privada Hilfr sobre salarios y condiciones de trabajo. Los trabajadores se benefician de un plan de pensiones, vacaciones pagadas y un seguro de enfermedad. En mayo de 2019, la empresa italiana de reparto de comida Laconsegna firmó un convenio colectivo con las tres federaciones sindicales mayoritarias que define a los riders como empleados. Los trabajadores están cubiertos por el convenio colectivo nacional para el sector logístico y se benefician de todas las protecciones sociales.

En Alemania, varias plataformas, junto con la Asociación de Crowdsourcing y el Sindicato de Metal IGMetall, han establecido una Oficina del Defensor del Pueblo particular para los trabajadores de las plataformas digitales para atender quejas y reclamaciones. Estas plataformas han firmado un código de conducta para crowdsourcing y crowdworking. En Colonia, los riders de Deliveroo, Foodora y Lieferando lograron establecer un comité de empresa con el apoyo del sindicato de alimentación y hostelería NGG en febrero de 2018. Un grupo de WhatsApp y un sitio de Facebook (https://www.facebook.com/liefernamlimit/) sirvieron como coordinación y medio de comunicación. Deliveroo impugnó la iniciativa con la no renovación de los contratos para todos los miembros del comité de empresa. Sin embargo, la lucha por la representación de los trabajadores continúa: Delivery Hero (Foodora), por ejemplo, ha tenido que aceptar representantes de los trabajadores en su consejo de supervisión de acuerdo con la ley alemana de codeterminación.

Ante las dificultades con la negociación colectiva en el sector, algunos sindicatos están tratando de incluir el trabajo de plataformas en convenios marco regionales o sectoriales. En Cataluña, los sindicatos y las organizaciones empresariales firmaron en julio de 2018 el Acuerdo Interprofesional de Cataluña (AIC) para 2018-2020, que incluye 
una sección sobre el trabajo de las plataformas y define explícitamente la relación entre una plataforma y un proveedor de servicios como relación laboral.

Fuera de Europa, también se están realizando las primeras experiencias con la organización colectiva de los trabajadores de plataformas. En Argentina, se fundó la Asociación Personal de Plataformas (APPSindical; @AppSindical) en 2018. En julio de 2019, el Tribunal Nacional de Justicia obligó a la plataforma de reparto Rappi a readmitir a los riders despedidos por su actividad sindical.

Aparte de estas formas incipientes de auto-organización y sindicalización, se han conocido también algunas experiencias con huelgas y acciones colectivas. En Bélgica, los riders pueden afiliarse a un sindicato y han establecido una plataforma auto-organizada de riders basado en la red (https://www.facebook.com/collectif.coursiers/). Cuando Deliveroo cambió unilateralmente su modelo de contrato de un régimen de empleado a un modelo de autónomo en 2017, varios colectivos de riders afectados, principalmente en Bruselas, organizaron huelgas y la ocupación temporal del edificio de Deliveroo. Aunque las acciones interrumpieron durante un tiempo el servicio de comida a domicilio, Deliveroo no alteró su modelo de contrato ni hizo ninguna concesión a los huelguistas (Vandaele et al. 2019).

En octubre de 2016, los trabajadores de Foodora en Turín organizaron huelgas y campañas públicas contra la conversión de sus contratos de contratos con pago por hora trabajada en contratos de pago por servicio realizado (Animento et al. 2017; https://www.facebook.com/DeliveranceProject/). Además de interrumpir los servicios, las campañas públicas y las manifestaciones de protesta dañaron la imagen de la plataforma y dieron como resultado un ligero aumento salarial, aunque se rechazaron todas las demás reivindicaciones de los huelguistas. Los contratos de todos los activistas de la huelga no se renovaron y la mayoría de ellos trabaja ahora para otras plataformas. Probablemente el impacto más importante de la huelga fue el aumento de la conciencia pública, que condujo a iniciativas parlamentarias para regular el sector de reparto y transporte.

En España, los trabajadores de Deliveroo y Glovo en Barcelona y Madrid crearon la plataforma 'Riders X Derechos' para reclamar su estatus de empleado y conseguir mejores condiciones de trabajo como, por ejemplo, un número garantizado de encargos por hora para tener unos ingresos más estables. En el verano de 2017, estos riders organizaron varias manifestaciones de protesta, reivindicaciones y huelgas puntuales que fueron contestadas con represiones y despidos. Para obtener apoyo logístico y asesoramiento jurídico en estos conflictos laborales, los trabajadores se pusieron en contacto con el sindicato regionalista catalán Confederación Sindical Alternativa de Cataluña. Cuatro riders de Deliveroo explican su situación (https://www.eldiario.es/hojaderouter/tecnologia/deliveroo-economia_colaborativa-riders_x_derechos-ciclomensajero-stuart-glovo_0_660034062.html):

"Te venden la moto de que eres libre para trabajar cuando quieras, pero en realidad estás supeditado a la forma que tiene cada empresa de repartir los horarios y el problema es que cada vez hay más repartidores y no hay volumen para todos."

"Venden la idea de que su negocio gira en torno a la tecnología, pero todo esto son pantallas de humo que esconden nuevas formas de explotación laboral. Mantenernos en régimen de autónomos y con bajos ingresos les conviene porque contribuye a que no haya sentimiento de grupo, a que compitamos entre nosotros por las horas y siempre haya un repartidor disponible".

"Si tienes algún problema puedes escribirles un correo, pero no hay teléfono de contacto. Puedes presentarte en la oficina, pero si no has pedido cita antes -nunca te la dan-, ni te abren la puerta."

Un rider de Deliveroo en Madrid que había participado en una demanda judicial contra la empresa explica las formas de represión: "Antes de mi ponencia con los diputados trabajaba 30 horas a la semana. Después la plataforma me las redujo a cuatro, sin explicación alguna. Es su nueva estrategia. En vez de desconectarte, si te quejas te reducen las horas de trabajo hasta hacerlo inviable" (El País, 23/02/2020).

En mayo de 2019, cientos de conductores de Uber en el Reino Unido se declararon en huelga como parte de una protesta internacional iniciada en varias ciudades de América del Norte. Exigían ser reconocidos como empleados y cobrar el salario mínimo (The Guardian, 08/05/2019). La acción fue apoyada por el Sindicato Independiente de Trabajadores de Gran Bretaña (IWGB).

Las condiciones específicas de empleo de los conductores y repartidores de las plataformas implican unas características particulares de su acción colectiva. En primer lugar, el contenido de las negociaciones y convenios es muy básico, aborda los derechos laborales más elementales, como los contratos de empleo regulares, los salarios mínimos, una jornada laboral estable, el derecho a vacaciones, el pago en case de enfermedad y la seguridad social. Los trabajadores de plataformas tienen que empezar a reivindicar sus derechos ahí donde comenzó el movimiento obrero en el siglo XIX. Whatsapp es el principal medio de comunicación y coordinación cuando se convoca una huelga, mientras que Facebook es el principal medio de organización y relaciones públicas. Las huelgas son cortas y puntuales, concentradas en las principales horas de entrega y acompañadas de campañas públicas contra la imagen de las plataformas. Atacar la marca de las plataformas y presionar a las autoridades públicas para que controlen y regulen el sector son los objetivos principales, dada la debilidad estructural de los activistas que a menudo sufren el despido en la forma de la no renovación de sus contratos.

La mayoría de los conflictos laborales de las plataformas se refieren a la situación contractual de los trabajadores. Los empleadores intentan esquivar las relaciones laborales convencionales, explotando la falta de claridad de la le- 
gislación laboral y obligan a sus trabajadores a un régimen de autónomos. Las normativas en la Unión Europea varían de un Estado a otro, algunos Estados miembros optan por la existencia de una relación laboral, mientras que otros apoyan la idea de que los trabajadores de la plataforma sean autónomos independientes. También existen discrepancias dentro de los Estados miembros, donde diferentes tribunales nacionales han dictado sentencias contradictorias (como en Francia, el Reino Unido y España) (Aranguiz 2019). Con respecto al caso de Uber, el Tribunal de Justicia de la Unión Europea dictaminó que Uber realiza un servicio de transporte clásico y que la relación legal con sus conductores debe considerarse una relación laboral (http://curia.europa.eu/juris/ liste.jsf? num = C-434/15).

En España, tres sentencias judiciales recientes en Barcelona, Valencia y Madrid confirmaron que los repartidores de Deliveroo y Glovo tienen una relación laboral con la plataforma y deben considerarse como empleados, no como autónomos. Los tribunales desmantelaron la posición de Deliveroo de la supuesta autonomía de sus repartidores, argumentando que la plataforma ejerce un control constante sobre los repartidores, que es la única poseedora de la información necesaria para el negocio y que el verdadero medio de producción es la plataforma en sí misma, y no el teléfono móvil y la bicicleta. Según las sentencias, los repartidores realizan un servicio personal en condiciones organizadas y dirigidas por la empresa (Aranguiz 2019; Todoli-Signes 2018). Además, la empresa utiliza el sistema de geolocalización (GPS) para la vigilancia constante de cada trabajador, de cada entrega y de todos los kilómetros recorridos. Por lo tanto, el propietario de la plataforma tiene que ser considerado un empleador. Es importante notar que el demandante en este caso no era un rider o un sindicato sino la Seguridad Social sobre la base de una investigación detallada por parte de la inspección del trabajo.

Otros de los problemas clave aunque menos discutidos de los trabajadores de plataformas son su aislamiento social, la falta de voz y la falta de comunicación. A crowdworker ('Tasker') analiza su situación:

"Siento que estoy alienado, de alguna manera, porque al mismo tiempo, como si vas a algún lado y tienes que trabajar con personas, entonces todavía estás con alguien, y aquí termino completamente solo. Pero es como si sintiera que nada de lo que digo o hago tiene un impacto en la empresa en la que confío. Así que siento que se supone que es una plataforma, pero las reglas de la plataforma..., no tengo nada que decir sobre las reglas de la plataforma y mi impacto es cero" (Huws et al.2017, 42; traducción propia)

Hilary, una repartidora en el Reino Unido, expresó este sentimiento:

"La empresa en sí es extraña para trabajar, en el sentido de que... solo puede comunicarse con ellos por correo electrónico. Nunca he conocido a nadie oficialmente de [nombre de la plataforma] desde el primer día en que fui entrevistada... Hay un número de teléfono al que se puede llamar durante el turno si tienes un problema con la entrega o si tienes un problema con la aplicación, pero no está permitido llamar a ese número para hablar sobre cambios u otros problemas. Como a veces me pagaron mal, tuve que enviar un correo electrónico, y es un proceso bastante lento y puede ser bastante frustrante... incluso si es su culpa, no pagan hasta el próximo sueldo, y luego cometen otro error y, todo esto, tienes que ir a través de correos electrónicos, explicando nuevamente, a una persona diferente cada vez, cuál es la situació" (Huws et al.2017, 41).

Muchos trabajadores de plataformas están convencidos que esta falta de contacto personal directo conduce a un comportamiento negativo y una toma de decisiones arbitraria que normalmente sería inaceptable en las relaciones cara a cara con los gerentes. Los empleadores son invisibles, ocultos detrás de la aparente neutralidad de la tecnología del algoritmo. La información, las calificaciones, las evaluaciones, todas las comunicaciones son mensajes anónimos pseudo-objetivos, aunque en realidad son la consecuencia de la programación y de las decisiones de la gestión de la plataforma. El aislamiento social y la alta intensidad de trabajo bajo la presión de las calificaciones y el control algorítmico son problemas reportados por ambos colectivos de trabajadores: repartidores locales y proveedores de servicios globales como Amazon Mechanical Turk o Upwork (Wood et al. 2019; Pesole et al. 2018 ; Perera et al. 2020). Como los trabajadores no tienen influencia sobre el contenido de las aplicaciones, se les deja sin ningún control sobre sus medios de producción.

En varios países ha surgido otro tipo de agente social de la economía de plataformas: las cooperativas que organizan a los trabajadores por cuenta propia y les brindan una serie de servicios profesionales. Una de las más establecidas es SMart (Société Mutuelle pour Artistes), una organización fundada en Bélgica en 1998 como una asociación de trabajadores independientes creativos y culturales y luego transformada en una cooperativa sin fines de lucro (Vandaele et al. 2019). SMart opera actualmente en nueve países europeos y se ha extendido a otros sectores más allá del trabajo creativo. A cambio de una tarifa, brinda a los trabajadores independientes una amplia gama de servicios, incluida la ayuda con la facturación y la declaración de la renta, el pago como empleado (y, por lo tanto, el acceso a la seguridad social), el cobro de deudas y los anticipos de pago a través de un fondo de garantía mutua y acceso a cursos de formación y de trabajo compartido.

SMart se basa en un proceso participativo: todos los miembros están invitados a participar en la asamblea general anual, y todas las ganancias se reinvierten. SMart, al igual que otras cooperativas de trabajadores similares, no suele negociar en nombre de sus miembros. En alguna ocasión expresa públicamente las preocupaciones y reivindicaciones de los trabajadores independientes y actúa en su nombre, pero este no es su objetivo principal. Algunos sindicatos han criticado el modelo propuesto por SMart por "legitimar las zonas grises" en lugar de luchar contra ellas (Xhauflair et al. 2017). 
En España la plataforma 'Riders X Derechos' lanzó en otoño de 2018 su propia cooperativa 'Mensakas' ('App de comida a domicilio responsable') para crear empleos decentes y luchar contra la precariedad.

Mensakas es una app de pedido online y entrega a domicilio. Este abril ya hemos constituido la cooperativa Mensakas SCCL que será autogestionada por la gente que trabaja y donde todo el mundo tendrá contrato laboral y cotizará debidamente a la seguridad social. Queremos ofrecer así una opción para que todo el mundo pueda consumir de forma socialmente responsable a través de su smartphone. El proyecto de la app de Mensakas fortalecerá la autoorganización entre riders para seguir luchando contra la precariedad, y es una experiencia de colectivización y soberanía económica. Con los medios digitales, logísticos y mediáticos que disponemos contribuiremos a impulsar la economía social y solidaria. Nos desplazaremos de forma totalmente ecológica y sostenible por la ciudad porque sólo utilizaremos bicicletas y vehículos eléctricos no contaminantes. (https://www.goteo.org/project/mensakas--app-de-menjar-a-domicili-responsable)

Mensaka se afilió a la federación europea de cooperativas de entrega en bicicletas CoopCycle (https://coopcycle. org/en/) fundada en 2016 y operativa en 16 ciudades europeas. En América del Norte, cientos de cooperativas de plataformas ya están tratando de desarrollar una alternativa de trabajo decente y democrático, basada en la propiedad de sus socios, para la creciente 'economía gig' (Hayes 2019).

La necesidad de regular la economía de plataformas y garantizar los derechos fundamentales de los usuarios, trabajadores y ciudadanos no solo la sienten los empleados, sino también un número creciente de ayuntamientos de grandes ciudades. Durante la Cumbre Sharing Cities en Barcelona del 12 al 15 de noviembre de 2018, 31 ciudades de todo el mundo, incluidas Ámsterdam, Barcelona, Lisboa, Madrid, Montreal, Nueva York, París, Sao Paulo, Seúl, Milán y Viena, firmaron la 'Declaración de Principios y Compromisos de las Ciudades Colaborativas' para reclamar su soberanía al negociar con grandes plataformas digitales que impactan negativamente en sus economías urbanas, como ha sido el caso con Airbnb y Uber. La búsqueda de modelos sostenibles y socialmente responsables de la economía de plataformas para las grandes áreas urbanas puede convertirse en un aliado natural para los empleados del sector en su lucha por los derechos laborales.

Como la economía de plataformas va en aumento y está en gran medida compuesta por trabajadores jóvenes con niveles educativos altos y familiarizados con las nuevas tecnologías de comunicación, se puede esperar que surjan organizaciones colectivas y formas innovadoras de representación de intereses a pesar de las condiciones laborales adversas. Las formas de organización y las formas de acción colectiva a desarrollar aún son bastante abiertas, algunas revelando similitudes con los primeros días del sindicalismo en el siglo XIX. Hay opiniones divididas entre aquellos que se orientan hacia los sindicatos tradicionales y aquellos que piensan en organizaciones alternativas de interés auto-organizadas o entre aquellos que luchan por los derechos laborales en las plataformas actuales y los que están a favor de crear plataformas alternativas más orientadas al cooperativismo. "Los patrones emergentes actuales sugieren una posible coexistencia o combinaciones de sindicatos convencionales y otros sindicatos nuevos y organizaciones similares que defienden las necesidades e intereses específicos de los trabajadores de plataformas" (Vandaele 2018, 6; traducción propia).

\section{Las fuentes de poder de los trabajadores de plataformas}

Para evaluar de manera más sistemática el potencial de acción colectiva y de organización de los trabajadores de plataformas, nos referimos al enfoque de las fuentes de poder desarrollado y empleado en varias investigaciones recientes sobre los sindicatos en Europa (ver Gumbrell-McCormick y Hyman 2013; Lehndorff et al. 2018; Vandaele 2018). Las organizaciones de trabajadores tienen cuatro fuentes de poder tradicionales, desarrollados en las luchas y los conflictos a lo largo de los siglos XIX y XX:

i. estructural: poseer cualificaciones o competencias escasas u ocupar posiciones estratégicas en el proceso productivo, otorgando al sindicato poder de negociación en la empresa o en el mercado;

ii. asociativa: afiliación, disposición a pagar, proporcionar recursos financieros al sindicato;

iii. organizacional: solidaridad y unidad para apoyar colectivamente su propósito y sus políticas, disposición para actuar (militancia);

iv. institucional: apoyo legislativo, administración del bienestar social, concertación tripartita (corporatismo).

Con respecto a las posibles estrategias de revitalización sindical y el desarrollo de formas innovadoras de resistencia contra las nuevas formas de explotación y precarización en tiempos de crisis y debilidad, estas fuentes de poder tradicionales requieren tres recursos complementarios y más discursivos que no son necesariamente nuevos, aunque pueden haber sido ligeramente olvidados o insuficientemente apreciados en el pasado:

a. moral: una misión e identidad basada en la lucha a favor de la justicia social y una sociedad mejor;

b. de colaboración o coalición: buscar aliados y compartir recursos con otros grupos y movimientos que tienen objetivos e intereses en común; 
c. estratégico: uso inteligente y más efectivo de recursos escasos.

El poder estructural de los trabajadores de plataformas es bajo debido a su fácil sustitución, los bajos requisitos de cualificación y su posición desorganizada en el proceso productivo. Esta debilidad se refiere al menos a los trabajadores de transporte y reparto. La situación puede ser algo diferente para trabajadores independientes altamente calificados en industrias creativas como diseño gráfico, desarrollo de páginas web y de software, edición, traducción, etc., pero estos tampoco suelen recurrir a la organización sindical para la defensa de sus intereses.

Su poder asociativo también es bajo debido a las evidentes dificultades para unir a trabajadores dispersos sin una relación laboral clara en organizaciones de afiliación estables y estructuradas.

Su poder organizativo es más fuerte de lo esperado inicialmente. Aunque organizarse es difícil sin un centro de trabajo común y experiencias laborales compartidas, la cultura de comunicación de las plantillas jóvenes, a menudo con elevados niveles educativos, a través de las redes sociales y los contactos informales facilita en muchos casos respuestas coordinadas a los abusos de los empleadores, y fomenta prácticas de auto-organización, de formar comunidades en red como los grupos Whatsapp, campañas y huelgas espontáneas. Sin embargo, las relaciones de poder siguen siendo muy asimétricas, ya que las plataformas pueden simplemente desconectar o excluir repartidores conflictivos y ajustar sus mecanismos de control algorítmico.

El poder institucional de los trabajadores de plataformas es casi inexistente ya que ellos trabajan fuera de las estructuras sindicales y de representación tradicionales y no son reconocidos como un colectivo con derechos establecidos en el sistema institucional público. Solo en algunos casos excepcionales, como los repartidores de Foodora en Alemania que envían representantes al consejo de vigilancia de la empresa o sus colegas austriacos que tienen reconocido su comité de empresa, los trabajadores de las plataformas han podido beneficiarse de las instituciones de representación existentes. Pero incluso en estas situaciones excepcionales, su poder institucional es muy limitado, ya que las plataformas pueden trasladar fácilmente su sede a otro país.

En esta situación de debilidad de las fuentes convencionales de poder sindical, las fuentes de poder discursivas complementarias, como las de poder moral y colaborativo, cobran una particular importancia. A medida que los ciclistas son visibles en las calles de la ciudad y establecen relaciones colaborativas con los restaurantes proveedores, las estrategias como las campañas públicas, las protestas y las acciones en red contra las marcas pueden convertirse en fuentes de poder efectivas en manos de los trabajadores de plataformas. No solo los propietarios de las plataformas, sino también las autoridades públicas y los tribunales de justicia sienten la presión de participar activamente en la defensa de los derechos laborales y sociales de este nuevo colectivo. Ejemplos como la Riders Union Bologna, una red de repartidores de comida que se declararon en huelga el 23 de febrero de 2018, obligando así a las plataformas a suspender sus servicios, muestran el potencial del poder discursivo. El 31 de mayo de 2018, este colectivo logró firmar una "Carta dei diritti fondamentali del lavoro digitale nel contesto urbano" (Carta de derechos básicos del trabajo digital en un contexto urbano) con las tres principales confederaciones sindicales, el ayuntamiento de centro izquierda y algunas plataformas locales de reparto de comida. En una acción de denuncia pública, tanto las plataformas firmantes como las no firmantes aparecen en la página web de la ciudad. Otras ciudades han expresado su interés en seguir el ejemplo de Bolonia (Vandaele 2018, 17). Los sindicatos alemanes del sector metalúrgico y de alimentación, bebidas y hostelería han adoptado un enfoque similar al promover un Código de Conducta de Crowdsourcing para ser firmado por las plataformas.

El ejemplo de la huelga de Foodora en agosto/septiembre de 2019 en Oslo ilustra los diferentes recursos de poder movilizados. Un grupo de Foodora riders organizados en el "Club Foodora" se afilió al Sindicato de Trabajadores del Transporte para exigir un convenio colectivo con mejoras salariales y órganos de representación regulares (delegados sindicales y un comité de empresa). Cuando la dirección de Foodora contestó que las demandas eran inaccesibles, unos 200 repartidores se declararon en huelga, organizando varias semanas de coloridas manifestaciones en bicicleta en las calles de Oslo ('pink parades'), campañas en las redes sociales, un 'comedor popular' de los riders de Foodora , etc. Al hacerlo, recibieron el apoyo público no solo de la confederación sindical LO sino también de partidos políticos, políticos locales, círculos académicos y medios de comunicación. El éxito de la huelga, un convenio colectivo que garantiza salarios y condiciones de trabajo dignos e incluye una representación institucionalizada y órganos de negociación (comité de empresa), muestra la efectividad de combinar las fuentes de poder disponibles en el contexto noruego. El mercado laboral local obliga a los empleadores a pagar salarios competitivos y otorgar a los trabajadores el estatus de empleados, una forma de poder estructural ausente para la gran mayoría de los trabajadores de plataformas en otros países. El poder establecido de los sindicatos y los derechos laborales avanzados agregan un importante poder institucional y asociativo, utilizado de forma eficaz por un grupo de líderes militantes (poder organizacional). Finalmente, los huelguistas pudieron movilizar el apoyo público y la visibilidad, presionando a la empresa y a las autoridades públicas para que escucharan sus demandas (poder discursivo). Esta disponibilidad excepcional de las principales fuentes de poder sindical fue capaz de superar el aislamiento, la precariedad y la falta de organización colectiva y de poder de negociación tan características para este sector.

Las plataformas de transporte, entrega de comida y cuidado muestran ciertas fuentes emergentes de poder discursivo y asociativo (Aloisi 2019). Los trabajadores de reparto con sus mochilas y vehículos de marca son visibles y, a menudo, se encuentran en zonas urbanas y puntos de espera, mientras que los trabajadores de cuidado a menudo establecen fuertes lazos personales con sus clientes. Estas condiciones permiten algunas formas embrionarias de solida- 
ridad, comunicación y apoyo público, especialmente porque estos trabajadores no son tan fácilmente reemplazables como otros crowdworkers. La denuncia pública de la marca, una práctica desarrollada originalmente por activistas de consumo alternativos, es una fuente de poder adicional en las manos de los trabajadores de plataformas, lo que abre oportunidades para nuevas coaliciones con otros movimientos cívicos en contextos urbanos.

\section{Conclusiones}

Considerando la creciente relevancia y el atractivo de la economía de plataformas en la economía global, en este artículo nos hemos concentrado en los potenciales y las prácticas emergentes de acción colectiva y la organización de los trabajadores de plataformas frente a las dificultades estructurales y en los intentos innovadores para contrarrestar la asimetría de los recursos de poder en este sector. La discrepancia entre las categorías y las regulaciones del empleo existentes y las prácticas laborales volátiles de las plataformas ha dejado a muchos trabajadores en una situación de desprotección y riesgo para su bienestar físico y social. Las autoridades políticas y los agentes sociales están llamados a actuar y abordar estos desafíos. "La aplicación de big data, nuevos algoritmos y el procesamiento de información en la nube cambiará la naturaleza del trabajo y la estructura de la economía. Pero la naturaleza exacta de ese cambio estará determinada por las decisiones sociales, políticas y económicas que tomamos"(Kenney y Zysman 2016; traducción propia).

El trabajo de plataformas es una nueva forma de re-comodificación del trabajo que carece todavía una definición clara, implica prácticas heterogéneas y algunas características comunes en términos de reorganización del trabajo y de modelos de negocio flexibles. "Forma parte de un conjunto de formas de trabajo justo a tiempo de alta volatilidad que se superponen y se basan en los medios digitales para su gestión" (Huws et al. 2017, 50; traducción propia). En este artículo nos hemos centrado en los trabajadores de reparto y de transporte, en sus intentos y potencialidades para organizarse y actuar colectivamente. Nuestro análisis de fuentes de poder ha revelado la importancia de la imagen pública y la marca de las plataformas como una base específica, sobre la cual se puede desarrollar la acción colectiva y la presión a favor del reconocimiento de los derechos laborales y sociales de los trabajadores de plataformas. Hasta qué punto el trabajo de plataformas representa una forma extrema de una tendencia general hacia la 'plataformización', 'uberización' o 'precarización' de nuestras economías (Aloisi 2019; Huws et al. 2017, 48; Standing 2011; Herod y Lambert 2016) sigue siendo una pregunta abierta, al igual que la posibilidad de encontrar nuevas formas de auto-organización colectiva fuera de los sindicatos tradicionales. Examinando los dos procesos, la difusión de estas prácticas de trabajo en los mercados laborales en general y el surgimiento de nuevas formas de acción colectiva flexible y auto-organización comunitaria en red, hemos encontrado evidencia empírica para su posible desarrollo en ambas direcciones.

\section{Bibliografía}

Aloisi, A. (2019), Negotiating the digital transformation of work: non-standard workers'voice, collective rights and mobilisation practices in the platform economy, EUI Working Paper MWP 2019/03, Badia Fiesolana: European University Institute.

Altenried, M. (2020), «The platform as factory: Crowdwork and the hidden labour behind artificial intelligence», Capital \& Class, vol. 44, pp. 1-14.

Animento, S.; Di Cesare, G. y Sica, C. (2017), «Total Eclipse of Work? Neue Protestformen in der Gig economy am Beispiel des Foodora Streiks in Turin», PROKLA, vol. 47 (2), pp. 271-290.

Aranguiz, A. (2019), «Spain à l'avant-garde on social protection for platform work? », Social Europe, 04/09/2019 (https://www. socialeurope.eu/spain-a-lavant-garde-on-social-protection-for-platform-work).

Blanco García, A. (2019), «Prospective future of platform cooperatives», Grassroots Economic Organizing, October 28, 2019, (https://geo.coop/articles/prospective-future-platform-cooperatives).

Coase, R. H. (1937), «The nature of the firm», ECONOMICA, vol. 4 (16), pp. 386-405.

Daugareilh, I.; Degryse, C. y Pochet, P. (eds.) (2019), The platform economy and social law: Key issues in comparative perspective, Bruselas: ETUI Working Paper 2019.10.

Degryse, C. (2016), Digitalisation of the economy and its impact on labour markets, Bruselas: ETUI Working Paper 2016.02.

Dueñas Herrero, L. J. (2019), «Retos y propuestas para la regulación del trabajo en los procesos productivos de las plataformas digitales», Cuadernos de Relaciones Laborales, vol. 37 (2), pp. 275-292.

Gumbrell-McCormick, R. and Hyman, R. (2013), Trade unions in Western Europe: hard times, hard choices, Oxford: Oxford University Press .

Harvey, D. (2003), The new imperialism, Oxford/N.Y.: Oxford University Press.

Hayes, R. (2019), «Worker-Owned Apps Are Trying to Fix the Gig Economy’s Exploitation», VICE Newsletter, Nov 19 (https:// www.vice.com/en_us/article/pa75a8/worker-owned-apps-are-trying-to-fix-the-gig-economys-exploitation).

Herod, A. y Lambert, R. (2016), «Neoliberalism, precarious work and remaking the geography of global capitalism», en Rob Lambert y Andrew Herod (ed.), Neoliberal Capitalism and Precarious Work, Cheltenham: Edward Elgar Publishing, pp. 1-36. 
Howcraft, D. y Bergvall-Kåreborn, B. (2019), «A typology of crowdwork platforms», Work, Employment and Society, vol. 33 (1), pp. 21-38.

Huws, U., Spencer, N.H., Syrdal, D.S. y Holts, K. (2017), Work in the European Gig economy: research results from the UK, Sweden, Germany, Austria, the Netherlands, Switzerland and Italy, Brussels and Hatfield: European Foundation for Progressive Studies, UNI Europa and University of Hertfordshire.

International Labour Organization (ILO) (2018), Digital Labour platforms and the future of work, Geneva: ILO.

Ivanova, M.; Bronowicka, J., Kocher, E. y Degner, A. (2018), Foodora and Deliveroo: The App as a Boss? Düsseldorf: Hans Böckler Stiftung, Working Paper Forschungsförderung $n^{\circ} 107$.

Kenney, M. y Zysman, J. (2016), «The Rise of the Platform Economy», Issues in Science and Technology, vol. 32 (3) (https:// issues.org/the-rise-of-the-platform-economy/).

Köhler, H.-D. y Martín Artiles, A. (2010), Manual de la Sociología del Trabajo y de las Relaciones Laborales, Madrid: Delta Publicaciones ( $3^{\mathrm{a}}$ ed. $)$.

Lehndorff, S.; Dribbusch, H. y Schulten, T. (2018), Rough waters. European trade unions in a time of crisis, Brussels: ETUI.

Luxemburg, R. (1912, 1978), La acumulación del capital, Barcelona: Grijalbo.

Mandl, I. (2019), Platform work: Maximising the potential while safeguarding standards?, Luxembourg: Eurofound.

Molina, O. y Pastor, A. (2018), «La Digitalización, Relaciones Laborales y Derecho del Trabajo», en Fausto Miguélez (coord.) La revolución digital en España. Impacto y Retos sobre el Mercado de Trabajo y el Bienestar. Bellaterra: Universitat Autònoma de Barcelona, pp. 311-342.

Perera K.; Ohrvik-Stott, J. y Miller C. (2020), Better Work in the Gig Economy, London: Doteveryone. https://doteveryone.org. uk/report/betterwork.

Pesole, A.; Urzí Brancati, M.C; Fernández-Macías, E.; Biagi, F. y González Vázquez, I. (2018), Platform Workers in Europe, EUR 29275 EN, Luxembourg: Publications Office of the European Union.

Standing, G. (2011), The Precariat: The New Dangerous Class, London: Bloomsbury Academic.

Sundararajan, A. (2015), «The 'Gig economy' is coming. What will it mean for work?», The Guardian, 26/07/2015.

Todoli-Signes, A. (2018), «Judgement designation Deliveroo 'rider' an employee and analysis of its impact on the 'Gig economy'», Transfer, vol. 24 (4), pp. 487-490.

Unión General de Trabajadores (UGT) (2019), El trabajo en las plataformas digitales de reparto, Madrid: Servicio de Estudios de la Confederación (serviciodeestudios@cec.ugt.org).

Vandaele, K. (2018), Will trade unions survive in the platform economy?, Brussels: ETUI Working Paper 2018.05.

Vandaele, K.; Piasna, A. y Drahokoupil, J. (2019), 'Algorithm breakers' are not a different 'species': attitudes toward trade unions of Deliveroo riders in Belgium, Brussels: ETUI Working Paper 2019.06.

Visser, J. (2019), Trade unions in the balance, ILO ACTRAV Working Paper: ILO.

Waters, F. y Woodcock, J. (2017), «Far From Seamless: a Workers' Inquiry at Deliveroo», Viewpoint Magazine, September 20, 2017 (https:/www.viewpointmag.com/2017/09/20/far-seamless-workers-inquiry-deliveroo/)

World Economic Forum (2020), Charter of principles for good platform work, http://www3.weforum.org/docs/WEF_Charter_ of_Principles_for_Good_Platform_Work.pdf.

Wood, A. J.; Graham, M.; Lehdonvirta, V. y Hjorth, I. (2019), «Good Gig, Bad Gig: Autonomy and Algorithmic Control in the Global Gig Economy», Work, Employment and Society, vol. 33 (1), pp. 56-75.

Xhauflair V., Huybrechts B. y Pichault F. (2018), «How can new players establish themselves in highly institutionalized labour markets? A Belgian case study in the area of project-based work», British Journal of Industrial Relations, vol. 56 (2), pp. 370-394.

Todos los enlaces y webs han sido accedidos el 29 de febrero de 2020. 